\title{
High coupling efficiency, passive alignment setup for visible-range fiber-to-waveguide edge coupling
}

\author{
Nathan C. Lin $\odot$, ${ }^{a}$ Sakib Hassan $\odot,{ }^{a}$ Xuan Zhao, ${ }^{\text {b }}$ Ashok Veeraraghavan, ${ }^{\text {a }}$ \\ and Jacob T. Robinson ${ }^{\mathrm{a}, *}$ \\ ${ }^{a}$ Rice University, Department of Electrical and Computer Engineering, Houston, \\ Texas, United States \\ ${ }^{\mathrm{b}}$ Rice University, Applied Physics Program, Houston, Texas, United States
}

\begin{abstract}
We present a method to passively edge couple multiple optical fibers with silicon nitride waveguides in the visible wavelengths. We efficiently convert the fiber mode to the waveguide mode using an inverse taper mode size converter and support passive alignment using a U-groove that centers the optical fiber to the inverse taper. In our prototypes, we measure a coupling efficiency of $-4.2 \mathrm{~dB}$ per facet. To reduce light leakage to the silicon substrate, we use a $6-\mu$ m oxide layer, which also eliminates the additional processes of undercutting the silicon substrate underneath the waveguide. Furthermore, the U-groove structure has a polished edge surface for coupling, reducing the steps of edge polishing the die. Fabrication of this visiblerange edge coupler is complementary metal-oxide-semiconductor-compatible, making it a highly scalable method for passively packaging multiple visible-range integrated photonics devices. (C) The Authors. Published by SPIE under a Creative Commons Attribution 4.0 Unported License. Distribution or reproduction of this work in whole or in part requires full attribution of the original publication, including its DOI. [DOI: 10.1117/1.JNP.14.046018]
\end{abstract}

Keywords: optical coupling; silicon nitride; silicon photonics.

Paper 20128 received Sep. 7, 2020; accepted for publication Dec. 18, 2020; published online Dec. 31, 2020.

\section{Introduction}

There is growing interest in silicon photonics devices due to their scalability and compatibility with the complementary metal-oxide-semiconductor (CMOS) processes. This growing interest is moving beyond telecommunication and data center sectors in the infrared wavelength, onto applications such as biosensing, optogenetics, and medical imaging in the visible wavelengths. ${ }^{1,2}$ For optogenetics, visible light is used to modulate neurons using light-activated proteins ${ }^{3-6}$ and to expose different engineered cell lines to release therapeutic protein or drugs in vivo. ${ }^{7-9}$ In addition, visible photonic platform can facilitate lab-on-chip spectroscopy, super-resolution microscopy, miniature endoscopy, and cytometry. ${ }^{10-13}$

A key aspect of using silicon photonics devices is coupling the light source from the fibers onto the nanometer-scale waveguides. Coupling methods are categorized into two groups: outof-plane vertical coupling with grating couplers and in-plane coupling with edge couplers. ${ }^{14,15}$ Grating couplers have a simpler fabrication process and higher alignment tolerance. However, they have a lower efficiency and narrower bandwidth. Edge couplers have large bandwidth and higher coupling efficiency, but the fabrication and assembling steps are more complex, involving polishing of the chip sides and high-precision alignment.

There has been tremendous research on edge coupling in the infrared range. In particular, a mode converter made with an inverse tapered waveguide is the most common edge coupling method to guide light from fiber onto the nanometer-sized waveguide with a low insertion loss. ${ }^{16-18}$ The inverse taper confines the large mode profile from the fiber onto the waveguide using a small effective index tip. Despite this advance, the packaging process often requires a time-consuming active alignment process to center the fiber to the inverse taper, which limits the scalability and increases the packaging cost.

\footnotetext{
*Address all correspondence to Jacob T. Robinson, jtrobinson@ rice.edu
} 
Passive alignment structure for edge coupling in the infrared range was first proposed by Park et al. ${ }^{19}$ They deep etched a U-groove for passive alignment to edge couple onto a polymerbased waveguide. Such design does not need mode conversion because the polymer waveguide dimension is in the micron range, similar to that of the fiber, with mode profiles of the same dimensions. ${ }^{19,20}$ Galan et al. ${ }^{21}$ later proposed to combine the passive alignment setup with a nanometer-scale silicon waveguide. They etched a V-groove using KOH (potassium hydroxide) in conjunction with an inverse taper silicon waveguide. However, precise dimension of the $\mathrm{V}$-groove is hard to control because $\mathrm{KOH}$ also etches the silicon oxide mask. ${ }^{21}$

The focus of this paper is on visible range edge coupling. Silicon nitride waveguide is used here because silicon absorbs light at the visible range. Silicon nitride can transmit a broad wavelength of light, from the visible to the infrared range. There has been a limited number of papers, specifically on such topic. Ong et al. ${ }^{22}$ designed and simulated a double-tip multimode interference coupler. There are some recent works on visible-range silicon photonics neural probes that implement edge and grating couplers. Mohanty et al. edge coupled a fiber onto a silicon nitride waveguide without the use of a mode size converter, and the device has an insertion loss of $16 \mathrm{~dB} .{ }^{3} \mathrm{Wu}$ et al. $^{23}$ and Shin et al. ${ }^{24}$ use U-groove for passive edge coupler alignment in conjunction with micrometer-dimension low refractive index waveguide to mode-match with the fiber. Their measured coupling losses are more than $10 \mathrm{~dB}$. Segev et al. ${ }^{25}$ used grating couplers to couple visible light into their neural probes for photostimulation, with a measured coupling loss of $\sim 16 \mathrm{~dB}$.

Here, we present a passively aligned edge coupler in the visible range with a low insertion loss. This work integrates the spot size converter design with U-groove passive alignment setup to facilitate the fiber-to-waveguide coupling process.

\section{Simulations}

A mode size converter is the main component of a low-loss edge coupler. It is composed of an inverse taper with a thin initial tip that gradually increases to the width of the waveguide. The small effective index of the taper tip delocalizes the light, forming a large mode profile that mode matches with the visible range fiber, which has a typical mode field diameter of $5 \mu \mathrm{m}$. To determine the optimal inverse taper length of the mode size converter, we used the Lumerical Eigenmode expansion (EME) solver to sweep through a range of inverse taper tip widths and taper lengths. Three wavelengths are simulated and shown in Figs. 1(a) and 1(b) for design guidance. In Fig. 1(a), the taper tip was swept to determine the power overlap efficiency of the single mode fiber and the inverse taper. Our design for the experimental 635-nm inverse taper device has an initial tip width of $90 \mathrm{~nm}$ that increased to $300 \mathrm{~nm}$ waveguide width to maintain the single mode confinement. In Fig. 1(b), the optimal taper length is simulated with taper length from 0 to $2000 \mu \mathrm{m}$, with the corresponding normalized transmission shown. The inset in Fig. 1(b) illustrates the shape of the inverse taper design. Our taper length is $1000 \mu \mathrm{m}$ for high transmission while maintaining a relatively small device footprint.

To demonstrate the mode conversion, we used the Lumerical 3D finite-difference timedomain (FDTD) simulation. The beam propagates from an SMF onto the mode size converter, as shown in the top-down view in Fig. 1(c). The corresponding mode intensity progression can be seen in the FDTD mode profiles in Figs. 1(d)-1(f). The large mode profile of the fiber was gradually confined into the waveguide.

\section{Fabrication}

The fabrication process was done on a 150-nm stoichiometric low-pressure chemical vapor silicon nitride layer on a 6- $\mu \mathrm{m}$ thick oxide, shown in Fig. 2(a). A thick layer of oxide was used to minimize light leakage from the SMF to the silicon substrate. Previously, Chen et al. ${ }^{26}$ performed a multiple-step process to selectively etch the silicon substrate at the bottom of the waveguide to form a cantilever structure. Such process was important because with the standard box layer of $2 \mu \mathrm{m}$, the beam leaks into the silicon substrate unless one uses a lensed fiber.

First, the tapered mode size converter was patterned using electron-beam lithography. The pattern was then transferred to the silicon nitride device layer by reactive ion plasma etching 
(a)

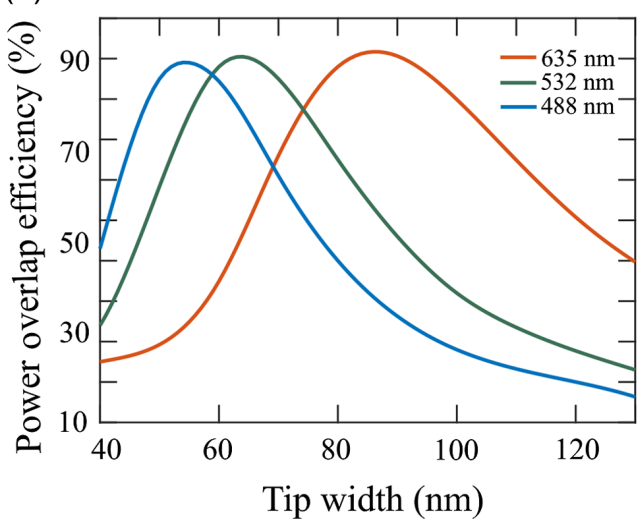

(b)

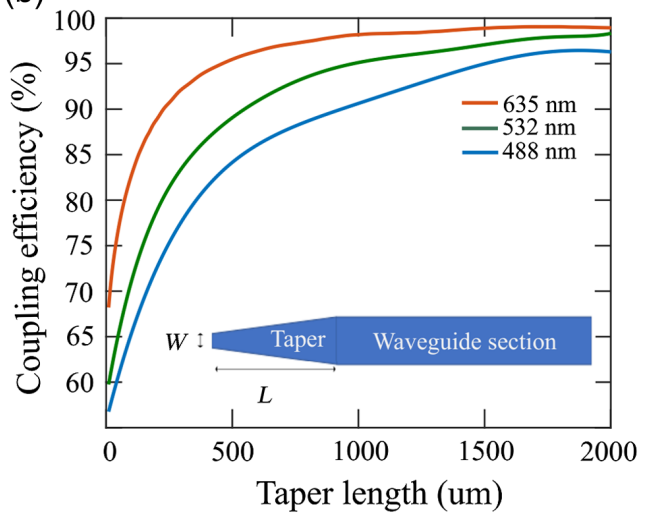

(c) $\quad 3=635 \mathrm{~nm}$

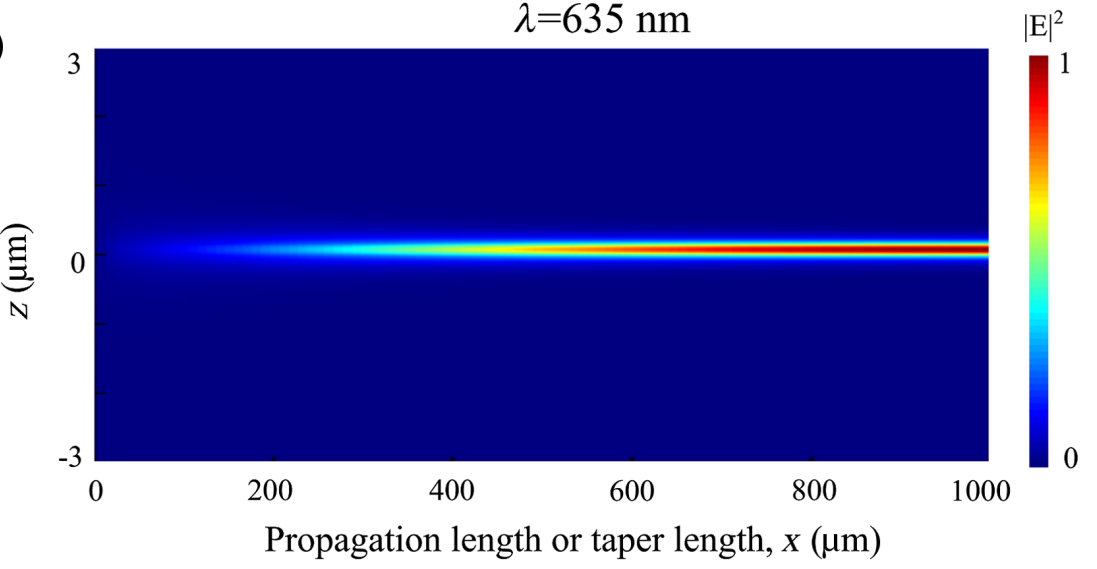

Mode profiles at different propagation length $(\lambda=635 \mathrm{~nm})$

(d)

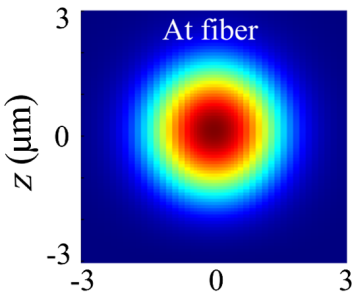

(e)

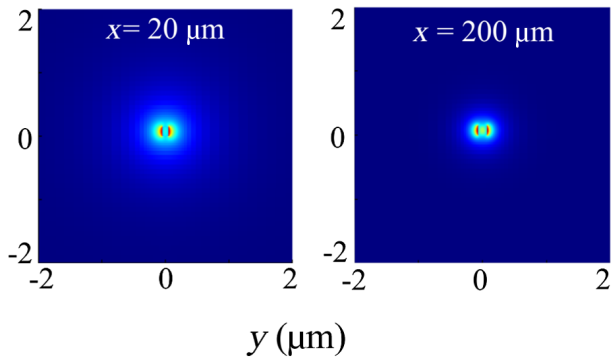

(g)

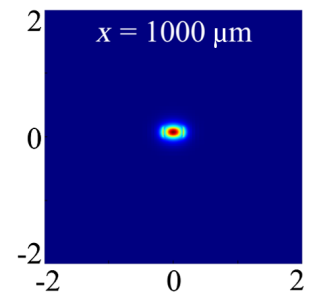

Fig. 1 (a) EME sweep of the inverse taper tip width and the corresponding power overlap efficiency. (b) EME sweep of the taper length from 0 to $2000 \mu \mathrm{m}$, with the corresponding coupling efficiency. The inset schematic illustrates the shape of the inverse taper design. (c) The top-down view of the mode profile progression as the mode size converter gradually confines the beam from the SMF. (d) The mode profile of the beam along the mode size converter at the fiber. (e) The mode profile at $x=20 \mu \mathrm{m}$. (f) The mode profile at $x=200 \mu \mathrm{m}$. (g) The mode profile at $x=1000 \mu \mathrm{m}$.

(RIE), shown in Fig. 2(e). Following that, U-grooves (125 $\mu \mathrm{m}$ wide and $6500 \mu \mathrm{m}$ long), centeraligned with the tapered waveguides with 200-nm gap in-between, were defined by a lift-off process with 250-nm-thick evaporated chromium hard mask, as shown in Fig. 2(f). Gold alignment markers were used for this multistep fabrication process. A 200-nm spacing was designed between the SMF and the waveguide to protect the waveguide during the U-groove etching process.

To create a U-groove depth of $62.5 \mu \mathrm{m}$ to fit the fiber, inductively coupled plasma-RIE with fluorine-based recipe was used to etch the 6- $\mu \mathrm{m}$-thick oxide layer, and SF6 recipes were used 
(a) $\mathrm{SiO}_{2}$ and $\mathrm{Si}_{3} \mathrm{~N}_{4}$ growth on $\mathrm{Si}$

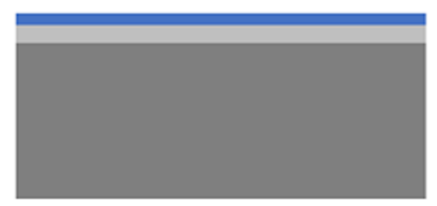

(b) Ebeam-patterning of alignment marks

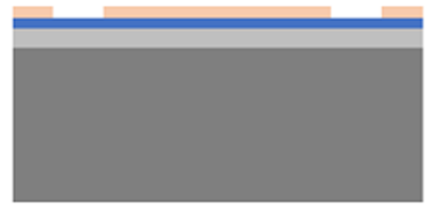

(c) Lift-off of alignment marks

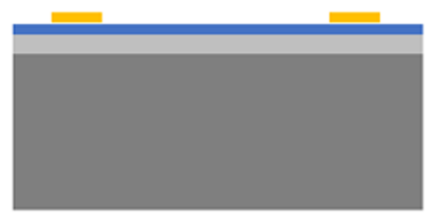

(d) Ebeam-patterning of waveguides

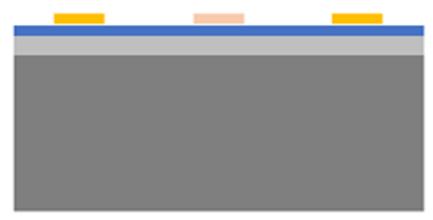

(e) RIE of waveguides

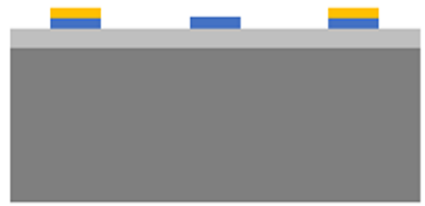

(f) Deposition of $\mathrm{Cr}$ hard mask

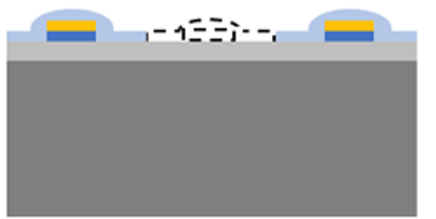

(g) DRIE of trenches

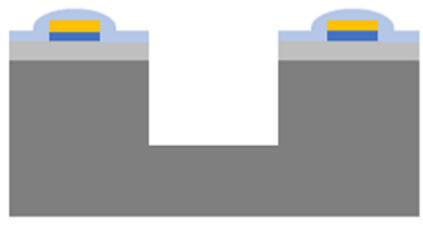

(h) Stripping-off of $\mathrm{Cr}$

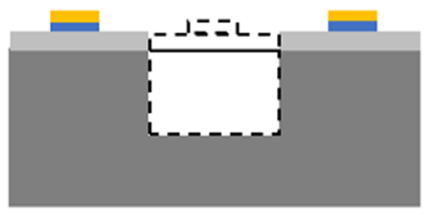

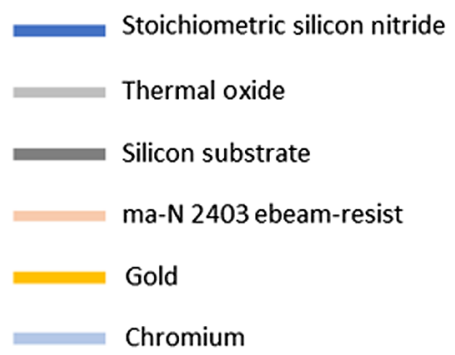

Fig. 2 (a) The wafer structure with 150-nm silicon nitride, $6 \mu \mathrm{m}$ of $\mathrm{SiO}_{2}$, and the silicon substrate layer. (b), (c) Gold alignments are placed with a lift-off process. (d), (e) The inverse tapered waveguide was defined using electron beam lithography and RIE etch. (f), (g) The U-groove was defined with a lift-off process and hard masked with chromium for deep etching. (h) The hard mask was stripped with chromium etchant.

to etch $56.5 \mu \mathrm{m}$ of the silicon substrate, as shown in Fig. $2(\mathrm{~g})$. To attain a high etch-depth precision, the Bosch process is first applied to etch the U-groove until $\sim 60 \mu \mathrm{m}$, followed by a slower silicon etch recipe using SF6 and oxygen at $200 \mathrm{~nm}$ per minute to get to $62.5 \mu \mathrm{m}$ depth. Across the sample, 200-nm depth variation was measured at different U-grooves due to the slight nonuniformity of the dry etching process. Ultimately, chromium was removed to uncover the silicon nitride waveguide, as shown in the dotted rectangle in the figure.

\section{Measurement and Discussion}

The fabricated device was characterized using a Fabry-Perot benchtop laser source with 635-nm wavelength (Thorlabs S1FC635) $2.5 \mathrm{~mW}$ power, visible range SMFs, CMOS camera (Imaging Source DFK 72BUC02), and a visible range power meter (Thorlabs PM100D with sensor 
S120C). The SMFs are aligned to the U-groove using three-axis nanostage manipulators (Thorlabs MAX313D), and ultraviolet (UV) epoxy (Norland Optical Adhesive NOA 85) is used to create a matching refractive index upper cladding before transmitting light through the waveguide. The UV epoxy has a transmission of $100 \%$ in visible spectrum range and it also seals off any gap between the fiber and the U-groove. The refractive index of the UV epoxy is $\sim 1.46$, matching to the thermal oxide bottom cladding layer; this allows the mode at the inverse taper tip to match with that of the fiber.

The U-groove structure has a polished edge surface, reducing the steps of edge polishing the die. The scanning electron microscope (SEM) image of the U-groove and the waveguide is shown in Fig. 3(a). The inset image Fig. 3(b) shows the magnified view of the U-groove and the waveguide. The edges of the U-groove are perpendicular to the wafer surface and have relatively smoother surfaces compare with the bottom because C4F8 gas was used to passivate the sidewall. The roughness of the bottom is measured to be $<100 \mathrm{~nm}$.

The experimental result is shown in Fig. 4. The input laser source is launched onto the device via a fiber from the right, and the device output is connected to a power meter through a fiber to the left, as shown in the camera image in Fig. 4(b). The diced sample has 18 U-grooves and the index-matching UV epoxy is used to secure the fibers. In Fig. 4(a), the top-down microscope image during the fiber alignment process into the U-groove is demonstrated. Figure 4(c) shows the top-down microscope image of the corresponding S-bend silicon nitride waveguide transmitting the 632-nm laser. The S-bend is to ensure that the light is guided by the waveguide, instead of traveling simply through the UV epoxy.

To determine the insertion loss of the edge couplers, we measured the end-to-end loss between the two edge-coupled fibers and propagation loss along the waveguide. Since the total end-to-end loss is the combination of insertion loss (at the input and output) and the propagation loss, we can compute the total insertion loss as the difference between end-to-end loss and the propagation loss. To measure the propagation loss of the waveguide, a microscope was used to acquire the scattering intensity as a function of the waveguide length for scattering analysis. ${ }^{27,28}$ The microscope was moved along the waveguide with a three-axis translational stage, and the
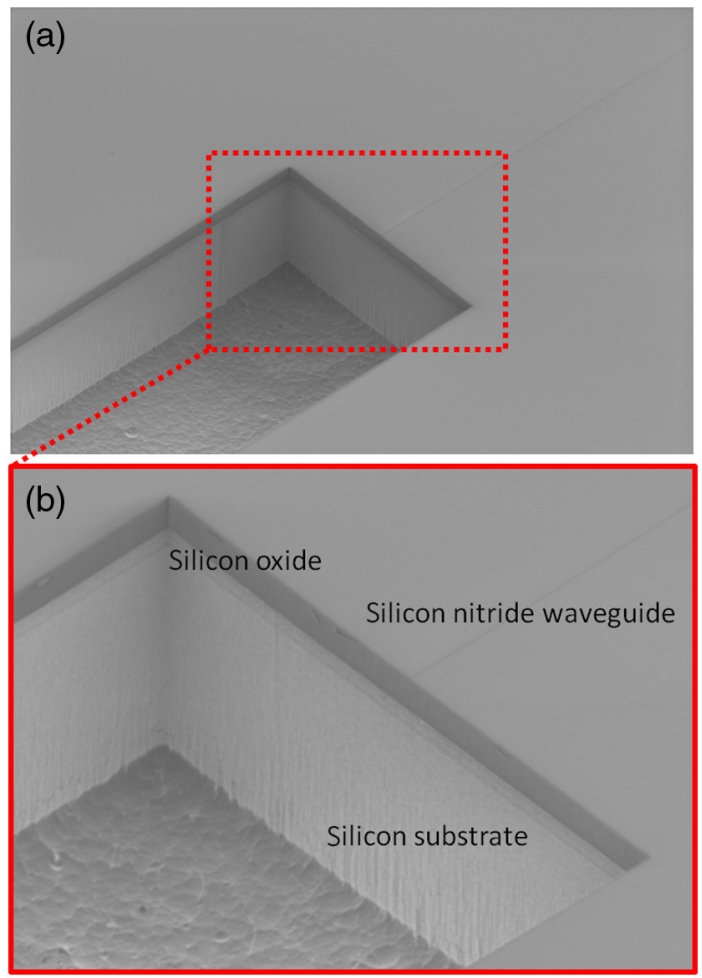

Fig. 3 The SEM image of a U-groove, with etched depth of $62.5 \mu \mathrm{m}$. (a) The U-groove is centeraligned with the inverse-tapered waveguide for optimal coupling. (b) The inset shows a magnified view of the U-groove and the waveguide. 


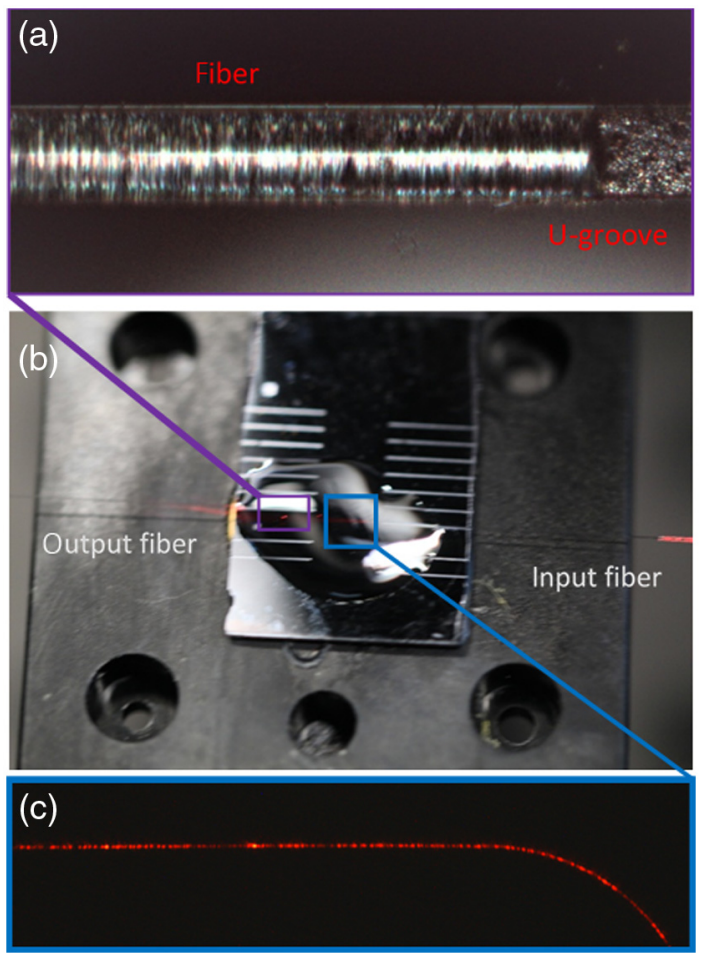

Fig. 4 (a) The top-down microscope image of the fiber alignment onto the U-groove. (b) Camera image of the setup. (c) The microscope image of the illuminated S-bend waveguide.

propagation loss was measured by continuously acquiring images of the illuminated waveguide along the propagation distance. The CMOS camera was set to have a longer exposure time to avoid pixel saturation. The scattering analysis result is shown in Fig. 5(a), where the relative intensity is referenced to the first image, and the propagation distance is $2100 \mu \mathrm{m}$. The measured propagation loss is $7.4 \mathrm{~dB} / \mathrm{cm}$ at $635 \mathrm{~nm}$ wavelength. The experimentally measured insertion loss of the edge coupler is then calculated from the end-to-end loss to be a value $-4.2 \pm 0.3 \mathrm{~dB}$ per facet based on measurements from six devices. The measured data are shown in Fig. 5(b).

This high coupling efficiency is demonstrated here not only because of the optimal alignment using the U-groove but also because of the high alignment capability of the electron-beam
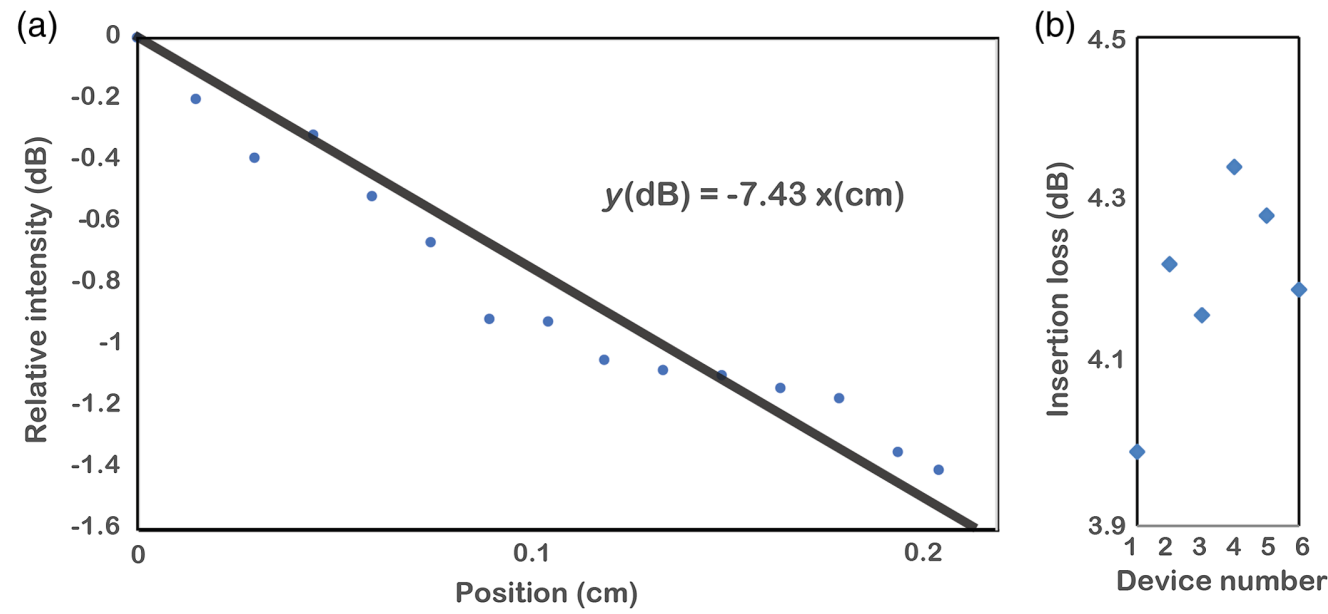

Fig. 5 (a) Scattering analysis is used to determine the propagation loss. The relative intensity of each data points represents the intensity at each image along the propagation distance. The relative intensity of each point is referenced to the first image closest to the input. (b) The measured insertion loss of the six devices. 
lithography system of $<20 \mathrm{~nm}$. With deep UV lithography system, the alignment difference could be up to $1 \mu \mathrm{m}$. Such difference would cause significant coupling loss due to light leakage and mode mismatch.

\section{Conclusion}

We demonstrated a passively aligned edge coupler in the visible range with a low insertion loss. The passive alignment reduces alignment time, eliminates the need to edge polish the sample, and allows for multiple optical fibers to edge couple with silicon nitride waveguides at wafer scale. This development allows for cost reduction and miniaturization of current surgical or medical imaging tools and facilitates the development of biosensing, optogenetics, and quantum optics. This passive alignment U-groove structure can be applied to a silicon-on-insulator setup for edge coupling in the infrared range.

\section{Acknowledgments}

This work was supported by DARPA under Award No. D20AC00002 and NIH under Award No. RF1-NS110501. The fabrication was conducted in part using resources of the Shared Equipment Authority at Rice University. We thank Dr. Tim Gilheart, Dr. Jeanne Guo, and James Kerwin at Rice University for their assistance. We also thank Dr. Long Chang at the University of Houston Nanofabrication Facility for his contribution. Disclosures: The authors declare that there are no conflicts of interest related to this article.

\section{References}

1. B. Desiatov et al., "Ultra-low-loss integrated visible photonics using thin-film lithium niobate," Optica 6(3), 380 (2019).

2. W. D. Sacher et al., "Visible-light silicon nitride waveguide devices and implantable neurophotonic probes on thinned $200 \mathrm{~mm}$ silicon wafers," Opt. Express 27(26), 37400 (2019).

3. A. Mohanty et al., "Reconfigurable nanophotonic silicon probes for sub-millisecond deepbrain optical stimulation," Nat. Biomed. Eng. 4(2), 223-231 (2020).

4. A. N. Zorzos, E. S. Boyden, and C. G. Fonstad, "Multiwaveguide implantable probe for light delivery to sets of distributed brain targets," Opt. Lett. 35(24), 4133 (2010).

5. E. S. Boyden et al., "Millisecond-timescale, genetically targeted optical control of neural activity," Nat. Neurosci. 8(9), 1263-1268 (2005).

6. E. Shim et al., "Multisite silicon neural probes with integrated silicon nitride waveguides and gratings for optogenetic applications," Sci. Rep. 6(1), 1-5 (2016).

7. J. Shao et al., "Smartphone-controlled optogenetically engineered cells enable semiautomatic glucose homeostasis in diabetic mice," Sci. Transl. Med. 9(387), eaal2298 (2017).

8. L. B. Motta-Mena et al., "An optogenetic gene expression system with rapid activation and deactivation kinetics," Nat. Chem. Biol. 10(3), 196-202 (2014).

9. M. A. Bochenek et al., "Alginate encapsulation as long-term immune protection of allogeneic pancreatic islet cells transplanted into the omental bursa of macaques," Nat. Biomed. Eng. 2(11), 810-821 (2018).

10. N. C. Lin et al., "Scanning optical coherence tomography probe for in vivo imaging and displacement measurements in the cochlea," Biomed. Opt. Express 10(2), 1032-1043 (2019).

11. A. Lichtenegger et al., "Spectroscopic imaging with spectral domain visible light optical coherence microscopy in Alzheimer's disease brain samples," Biomed. Opt. Express 8(9), 4007 (2017).

12. A. Rahim et al., "Expanding the silicon photonics portfolio with silicon nitride photonic integrated circuits," J. Lightwave Technol. 35(4), 639-649 (2017).

13. M. A. G. Porcel et al., "Silicon nitride photonic integration for visible light applications," Opt. Laser Technol. 112, 299-306 (2019). 
14. R. Marchetti et al., "Coupling strategies for silicon photonics integrated chips," Photonics Res. 7(2), 201-230 (2019).

15. L. Chrostowski and M. Hochberg, Silicon Photonics Design from Devices to Systems, Cambridge University Press, Cambridge (2015).

16. V. R. Almeida, R. R. Panepucci, and M. Lipson, "Nanotaper for compact mode conversion," Opt. Lett. 28(15), 1302-1304 (2003).

17. T. Shoiji et al., "Low loss mode size converter from $0.3 \mu \mathrm{m}$ square $\mathrm{Si}$ wire wave-guides to singlemode fibres," Electron. Lett. 38(25) 1669-1670 (2002).

18. G. Roelkens et al., "Efficient silicon-on-insulator fiber coupler fabricated using 248-nmdeep UV lithography," IEEE Photonics Technol. Lett. 17(12), 2613-2615 (2005).

19. S. Park, J.-M. Lee, and S.-C. Ko, "Fabrication method for passive alignment in polymer PLCs with U-grooves," IEEE Photonics Technol. Lett. 17(7), 1444-1446 (2005).

20. N. Keil et al., "PLC as an optical integration bench," in Opt. Fiber Commun. Conf. and Expos. and the Natl. Fiber Opt. Eng. Conf. 2011, Optical Society of America, Los Angeles (2011).

21. J. V. Galan et al., "Polarization insensitive low-loss coupling technique between SOI waveguides and high mode field diameter single-mode fibers," Opt. Express 15(11), 7058-7065 (2007).

22. J. R. Ong et al., "Silicon nitride double-tip fiber-to-waveguide edge couplers at visible wavelengths," in Presented at Conf. on Lasers and Electro-Opt. Pac. Rim (2017).

23. F. Wu et al., "An implantable neural probe with monolithically integrated dielectric waveguide and recording electrodes for optogenetics applications." J. Neural Eng. 10(5), 056012 (2013).

24. H. Shin et al., "Multifunctional multi-shank neural probe for investigating and modulating long-range neural circuits in vivo," Nat. Commun. 10(1), 3777 (2019).

25. E. Segev et al., "Patterned photostimulation via visible-wavelength photonic probes for deep brain optogenetics," Neurophotonics 4(1), 011002 (2017).

26. L. Chen et al., "Low-loss and broadband cantilever couplers between standard cleaved fibers and high-index-contrast $\mathrm{Si}_{3} \mathrm{~N}_{4}$ or Si waveguides," IEEE Photonics Technol. Lett. 22(23), 1744-1746 (2010).

27. J. Tinguely, O. I. Helle, and B. S. Ahluwalia, "Silicon nitride waveguide platform for fluorescence microscopy of living cells," Opt. Express 25(22), 27678 (2017).

28. S. M. Lindecrantz and O. G. Helleso, "Estimation of propagation losses for narrow strip and rib waveguides," IEEE Photonics Technol. Lett. 26(18), 1836-1839 (2014).

Nathan C. Lin joined Dr. Jin U. Kang's Optoelectronics Lab in 2012 as an undergraduate researcher at Johns Hopkins University. In 2013, he started his doctorate program in electrical engineering at Columbia University, studying with Professor Christine Hendon and Dr. Elizabeth Olson. There, he focused on building optical coherence tomography systems for bio-medical imaging. In 2019, he joined Dr. Jacob Robinson and Dr. Ashok Veeraraghavan's labs as a post-doctorate fellow to do silicon nanophotonics for biomedical sensing and imaging.

Sakib Hassan is a PhD student in the Electrical and Computer Engineering Department at Rice University, Houston, Texas, USA. He received his BSc degree in electrical and electronic engineering from Bangladesh University of Engineering and Technology and his MSc degree in electrical engineering from Rice University. His research focuses on developing integrated nanophotonic sensors for bio- and neuroengineering applications.

Xuan Zhao is a graduate student in the Applied Physics Department at Rice University, Houston, Texas, USA. His research interests are the areas of nanofabrication, integrated photonics, and neuroengineering. He received his BS degree in physics from Marietta College, Marietta, Ohio, USA, in 2017. He is a member of Sigma Pi Sigma and Phi Beta Kappa. He has served as a departmental representative of the Applied Physics Graduate Student Association at Rice University.

Ashok Veeraraghavan is a professor in the Electrical and Computer Engineering Department at Rice University. He received his BTech in electrical engineering from Indian Institute of 
Technology, Madras, in 2002, and his master's and PhD degrees from the Department of Electrical and Computer Engineering at the University of Maryland, College Park, in 2004 and 2008, respectively.

Jacob T. Robinson is an associate professor in the Department of Electrical and Computer Engineering with joint appointments in the Bioengineering Department at Rice University and the Neuroscience Department at Baylor College of Medicine. He received his BS degree in physics from the University of California, Los Angeles, in 2003, followed by his MS and PhD degrees in applied physics from Cornell University in 2008. From 2008 to 2012, he was a postdoctoral researcher in the Department of Chemistry and Chemical Biology at Harvard University, after which he joined Rice University as an assistant professor. His research is focused on developing scalable nanotechnologies to manipulate and measure brain activity. He is a recipient of the DARPA Young Faculty Award, the Materials Today Rising Star award, and a cochair of the IEEE Brain Initiative. 